\title{
Contribución a la logística inversa mediante la implantación de la reutilización por medio de las redes de Petri
}

\author{
Contribution to the inverse logistics be means of implantation \\ of the reuse through the Petri nets
}

\author{
L.O. Vega de la $\mathrm{Cruz}^{1 *} \quad$ C.E. Marrero Fornaris ${ }^{2} \quad$ M.C. Pérez Pravia ${ }^{3}$ \\ Recibido 7 de diciembre de 2015, aceptado 28 de abril de 2016 \\ Received: December 7, 2015 Accepted: April 28, 2016
}

\begin{abstract}
RESUMEN
Este artículo presenta una aplicación de las redes de Petri para la implantación de la logística inversa, mediante la reutilización. Siguiendo esta pauta se plantea la simulación de la reutilización de los envases en una línea de producción de helados, mediante el estudio del comportamiento dinámico del proceso, así como la validación de su comportamiento. Como resultado se destaca el perfeccionamiento de la reutilización en el proceso logístico de la empresa de servicios lácteos Rafael Freyre de Holguín, incrementando sus beneficios, como productividad y utilidades.
\end{abstract}

Palabras clave: Logística inversa, reutilización, modelado de procesos, redes de Petri, envases.

\begin{abstract}
This article shows an application of the Petri nets for the establishment of inverse logistics, by means of the reuse. Following this guideline the simulation of the reuse of containers in an ice creams production line comes into question, by means of the study of the dynamic behavior of the process, as well as the validation of its behavior. As a result, stands out the improvement of the reuse in the logistic process of the dairy services enterprise Rafael Freyre of Holguín, Cuba, increasing their benefits, such as productivity and utilities.
\end{abstract}

Keywords: Inverse logistics, reuse, modeling of processes, Petri's nets, containers.

\section{INTRODUCCIÓN}

Múltiples cambios en las relaciones comerciales, los efectos de las crisis capitalistas, las transformaciones tecnológicas y los adelantos científico-técnicos propician una significativa revolución en la organización de las actividades productivas y de servicios que han alcanzado niveles mundiales. Por consiguiente, las estrategias que siguen las empresas van encaminadas a mejorar su competitividad; lo que tiene un alcance que trasciende de su entorno inmediato, al proyectarse en la búsqueda y asimilación

1 Departamento de Ingeniería Industrial. Facultad de Ingeniería Industrial y Turismo. Universidad de Holguín "Óscar Lucero Moya”. Avenida XX Aniversario. Piedra Blanca, Holguín-Cuba. E-mail: leovega@uho.edu.cu

2 Departamento de Cuadros. Universidad de Holguín "Óscar Lucero Moya". Avenida XX Aniversario. Piedra Blanca, HolguínCuba. E-mail: cmarrero@uho.edu.cu

3 Facultad de Ingeniería Industrial y Turismo. Universidad de Holguín “Óscar Lucero Moya”. Avenida XX Aniversario, Piedra Blanca. Holguín, Cuba. E-mail: mpp@uho.edu.cu

* Autor de correspondencia. 
de las mejores prácticas, quienes garantizan un desempeño superior.

En el marco empresarial es posible precisar la existencia de operaciones como: transportación, manejo de materiales, almacenamientos, servicio a los clientes, procesamiento del pedido de los clientes, compras, gestión de información, entre otras. Según [1] la gestión logística de una organización es “...la acción del colectivo laboral dirigida a garantizar las actividades de diseño y dirección de los flujos materiales, informativo y financiero desde su fuente de origen hasta sus destinos finales que deben ejecutarse de forma racional y coordinada con el objetivo de proveer al cliente los productos y servicios en la cantidad, calidad, plazos y lugar demandados con elevada competitividad y garantizando la preservación del medio ambiente". Por lo que se considera acertado plantear que las actividades antes relacionadas constituyen actividades logísticas. La logística inversa aparece, en este contexto, como una solución a los incrementos de producción y a la creciente preocupación por la conservación medioambiental.

En los últimos años se ha producido un incremento en la generación de residuos y desechos: productos devueltos, ociosos, el cambio de equipos por tecnologías más novedosas en la industria, así mismo se incorporan cada vez más productos, partes o materiales recuperados de vuelta a los procesos productivos y mercados, por lo que cada vez más se necesita de empresas y cadenas que desempeñen esta labor eficientemente [2]. Este sistema de igual forma precisa de un eficiente control para su futura efectividad e intrínsecamente de esto una buena simulación de su comportamiento [2-4].

Con el propósito de ofrecer una mejor alternativa en la simulación de procesos productivos para implantar la reutilización, se recurrió a unas de las herramientas más utilizadas actualmente: las redes de Petri para el análisis de redes complejas [5-16], pero esta vez para el modelado procesos de producción. Estas permitirán detectar ausencias de situaciones críticas que afecten el adecuado desarrollo del proceso propuesto en el ámbito logístico, permitiendo así, la validación dinámica de los procesos y logro de su futura aplicación, además de permitir la simulación de los resultados previstos, posibilitando así incrementos fructíferos en un proceso productivo.

\section{MÉTODOS}

La práctica de la logística viene desarrollando actividades y funciones que quizás en el pasado resultaron suficientes. No obstante, en el contexto de negocios de altos volúmenes y bajos márgenes, se hace indispensable el desarrollo e implementación de nuevas formas de organización que permitan aumentar la productividad de los recursos de las empresas.

De esta forma, el nuevo entorno exige rapidez y flexibilidad ${ }^{4}$ en procesos productivos, implicando una nueva dinámica en la compra de materias primas necesarias en la manufactura, las que no son producidas al mismo ritmo que se consumen. De igual forma, sucede con los volúmenes de producción, quienes son mayores a las necesidades del mercado doméstico o regional, haciendo necesario llevarlos a otras regiones y países.

La consulta de los criterios de un grupo de especialistas en la materia [1,17], reafirman el análisis anterior y justifican cómo el alcance del concepto de logística empresarial ha avanzado en el tiempo evidenciándose su evolución como sistema e integración. Por lo que en este estudio se replantea lo dicho por los autores y valorando las variables más utilizadas en el análisis conceptual como se muestra en la Figura 1, se considera que la logística es el proceso de gestión ${ }^{5}$, de forma efectiva, del flujo de distribución-fabricación-distribución de los productos, materia primas y materiales, así como, el flujo de información desde el punto de origen hasta el consumo, con el objetivo de satisfacer las necesidades del cliente.

Por otra parte; la logística verde es el proceso de medir y reducir al mínimo el impacto ecológico de las actividades de la logística tradicional [18]. Todas las actividades descritas anteriormente en la logística directa, están presentes en la logística inversa, la diferencia estriba en que todas se realizan en sentido contrario, la logística verde también está relacionada con la logística inversa que ha sido objeto de análisis de muchos autores [2, 19-21]

\footnotetext{
4 La posibilidad que tiene de aplicarse a varias entidades con características no necesariamente idénticas.

5 Planificación, organización y control, este término ha sido trabajado por más de 300 investigaciones.
} 


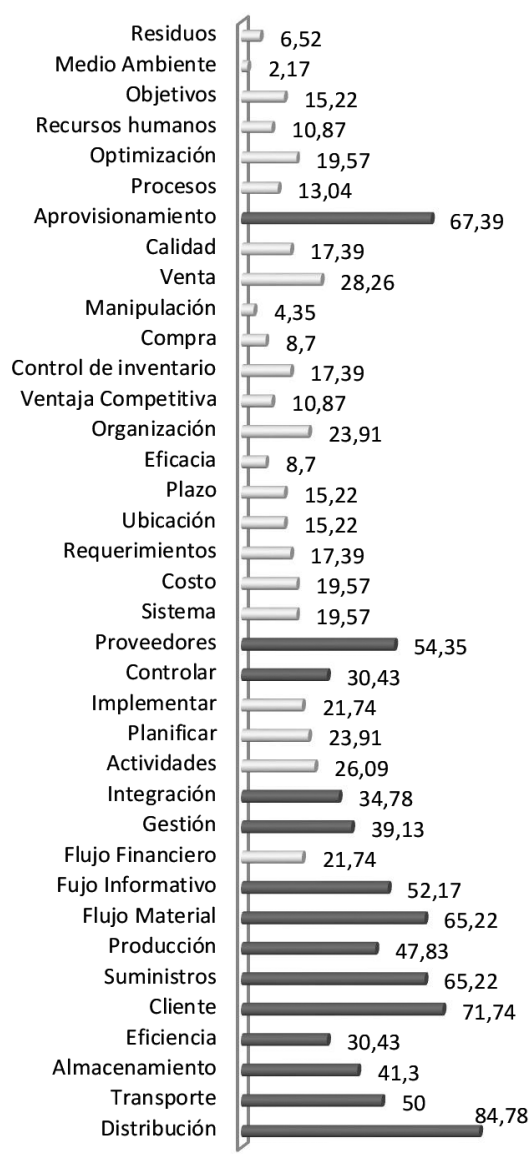

Figura 1. Análisis porcentual de las variables más tratadas en la concepción de la logística.

destacándose un conjunto de variables representadas en la Figura 2.

En la Figura 2 se representa las variables más utilizadas en la búsqueda conceptual, donde se observan tres grandes grupos: un primero representado por variables propiamente de la logística empresarial (transporte, gestión del flujo de producción, uso óptimo de recurso, etc.), el segundo representado por lo referido a la logística verde (gestión ambiental, consumo energético, reducción de residuos, etc.) y en el centro el grupo tres donde están las variables más relacionadas que son propias de la logística inversa (reventa, recuperación de valor, retorno al proveedor, etc.) de productos y envases. Dentro de la logística inversa se enfatizan:

Reprocesamiento: conjunto de actividades que añaden valor durante el proceso de transformación

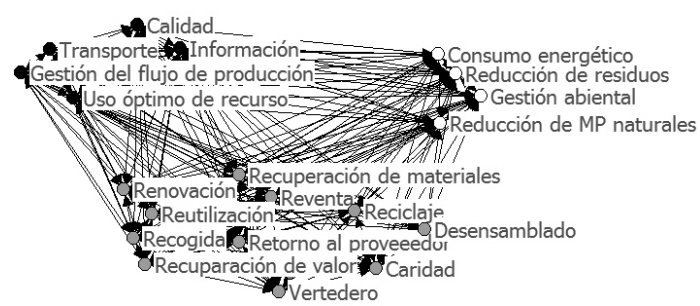

Figura 2. Relación de las variables más tratadas en la logística inversa.

convirtiendo los productos y partes retornados en productos reciclados;

Reciclaje: consiste en la recuperación del material contenido en los productos retornados mediante el desensamblado de las partes, su clasificación y su transformación en materias primas, es decir, implica nuevos procesos de producción;

Reutilización: los productos retomados pueden ser reusados (posiblemente después de limpiarlos o de una reparación menor) sin necesidad, de que sean introducidos en el proceso de producción;

Destrucción: aunque realmente no sería una alternativa válida de recuperación, este sería el último recurso en la eliminación de los productos al final de su vida útil.

Según lo antes establecido por los investigadores, en este estudio se considera que la logística inversa no es más que un sistema rediseñado para gestionar eficientemente el flujo de productos destinados al reprocesamiento, la reutilización, el reciclaje o la destrucción para solucionar los incrementos de producción y a la creciente preocupación por la conservación medioambiental.

La multitud de movimientos inversos de productos que pueden identificarse difieren en su función, mecanismo que los origina, el tratamiento que se hace de los mismos, etc. Resulta fundamental establecer claves de clasificación que permitan su consideración general desde la perspectiva de la gestión logística.

El flujo material de los residuos posee particularidades que deben ser estudiadas para lograr el diseño y la gestión de sus cadenas de suministro, buscando racionalidad, integralidad y efectividad. Estas variables posibilitan la aplicación de las filosofías de gestión logística, las cuales han llegado, incluso, a permitir la 
integración de varias organizaciones que persigan objetivos comunes para lograr la satisfacción de los clientes finales, facilitando así la toma de decisiones tácticas y operativas en los marcos de la cadena de suministro.

Según el análisis conceptual se destacan dos vertientes las realizadas con el producto y las realizadas con los envases y embalajes:

Producto: retorno al proveedor, reventa, caridad o mercados menos exigentes, reacondicionamiento, renovación o reprocesamiento por parte de la propia empresa o por parte de un tercero, recuperación de materiales, reciclaje y vertedero.

Envases y embalajes: reutilización, renovación, recuperación de materiales, reciclaje, caridad ${ }^{6}$ y vertedero.

Se profundizó en los enfoques metodológicos del sistema logístico, recibido en la literatura especializada nacional e internacional. Para realizar este análisis se utilizaron cerca de 90 investigaciones ${ }^{7}$ de diferentes latitudes del planeta (Figura 3).

El rango de fecha analizado transita desde 1997 hasta la actualidad, notándose que la mayor representatividad la poseen los años: 2003, 2004, 2006, 2007 y 2009.

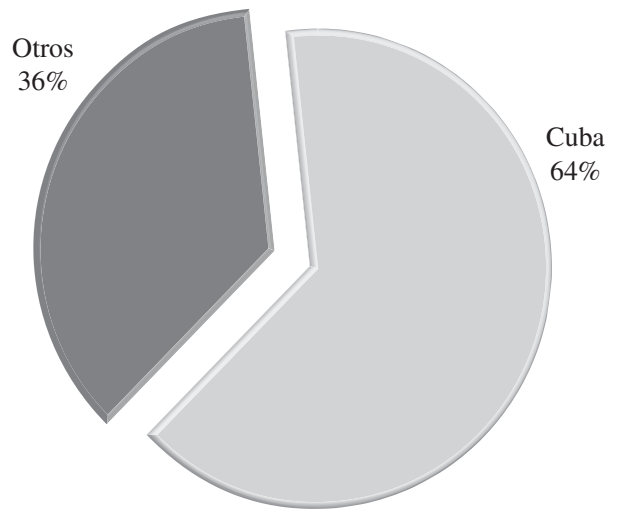

Figura 3. Investigaciones vs. latitud del planeta.

\footnotetext{
6 Misericordia, compasión, regalos, obra de gracia, etc.

7 De ellas 60 tesis doctorales de Cuba, América y Europa.
}

En la Figura 4 se observa, de forma detallada, este comportamiento. En la presentación por autores se pudo observar que se delimita la existencia de dos grupos relacionados con el sector de aplicación de las propuestas, siendo el primero el más grande conformado por aquellos que han trabajado en el sector terciario que representa el $52 \%$.

Las propuestas del resto son aplicadas al sector productivo de bienes, según la Figura 5.

El sistema logístico está compuesto por cuatro subsistemas: aprovisionamiento, producción distribución y reciclaje. Se analizó cuáles estaban presentes en las investigaciones (Figura 6), como resultado se observa que el subsistema que mayor tratamiento recibe es el de producción $(67,69 \%)$ destacándose investigadores como [1, 17], seguido por el de distribución $(63,08 \%)$ destacándose autores como [1, 17, 22] y aprovisionamiento [1, 17, 23] con un 55\%, En contraposición se encuentra el de reciclaje $36 \%$ como el menos abordado destacándose autores como [2, 19-21, 24-29].

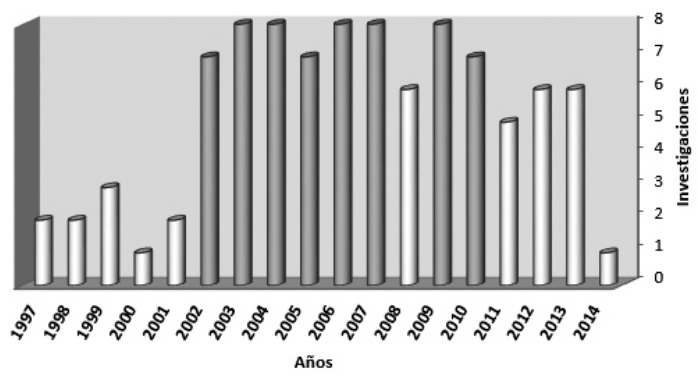

Figura 4. Cantidad de investigaciones por años.

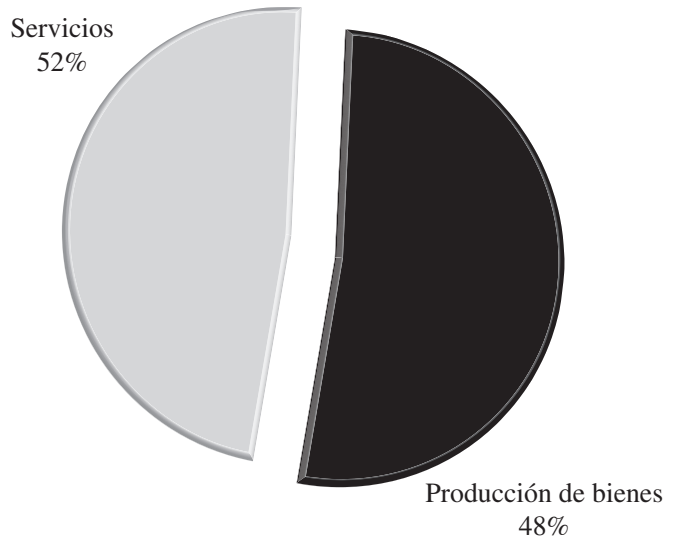

Figura 5. Investigaciones por tipo de sector. 


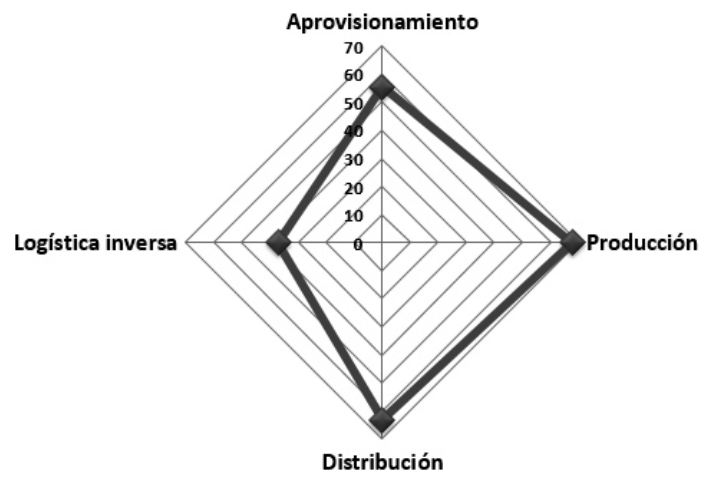

Figura 6. Porcentaje de investigaciones por subsistema logístico.

La matriz conformada para la comparación de las propuestas, en función de las características establecidas se analizó por medio de un análisis de segmentación donde en la Tabla 1 aparece la representación de la distribución de los enfoques analizados por sistema de producción, y el estudio exhaustivo de la logística inversa.

Tabla 1. Análisis de los enfoques de la logística inversa.

\begin{tabular}{|l|l|}
\hline Sistema productivo & \multicolumn{1}{c|}{ Reciclaje } \\
\hline Producción de bienes & $\begin{array}{l}\text { Llauró Fábregas (1999), } \\
\text { Knudsen González (2005), } \\
\text { Blanco Londono (2011) }\end{array}$ \\
\hline Servicios & $\begin{array}{l}\text { Cazull Imbert (2008), } \\
\text { Broche Fernández (2013) y }\end{array}$ \\
\hline \multirow{3}{*}{ Mixto } & $\begin{array}{l}\text { Hevia Lanier (2008), } \\
\text { Toledo Hernández (2010) y } \\
\text { Victoria de la Fuente (2009) } \\
\text { y Feitó Cespón (2016) }\end{array}$ \\
\hline
\end{tabular}

Del análisis anterior se concluye que una gran parte de los estudiosos de la logística inversa carecen de la utilización la modelación matemática, así como la simulación de los procesos, a diferencia de otros [2, 29] trabajan la modelación del proceso de logística inversa pero no lo detallan con el incremento de la producción mediante su flujo productivo, por lo que se concluye que no existe una investigación que modele el proceso de logística inversa y mejore e incremente los niveles de producción mediante el rediseño del proceso de la logística inversa.

\section{Análisis del método}

Se utilizó las redes de Petri (RdP), estas fueron utilizadas inicialmente para el análisis de algoritmos en la computación paralela o concurrente, pero dada la complejidad de los procesos productivos actuales, las RdP constituyen un método alternativo de diseño tanto para el proceso industrial como para el controlador $[5,11,15]$. Una RdP es un grafo orientado con dos tipos de nodos: lugares $(\mathrm{P})$ representados mediante circunferencias y transiciones (T) representadas por segmentos rectos verticales. Los lugares y las transiciones se unen mediante arcos o flechas (F). Un arco une siempre lugares con transiciones y viceversa, teniendo determinados pesos (W) (Figura 7). Los lugares pueden presentar marcas (una marca se representa mediante un punto en el interior del círculo) y necesitan de un marcado inicial $\left(\mathrm{M}_{0}\right)$. Formalmente, una $\mathrm{RdP}$ se define como una quíntupla, $\mathrm{RdP}=\left(\mathrm{P}, \mathrm{T}, \mathrm{F}, \mathrm{W}, \mathrm{M}_{0}\right)$.

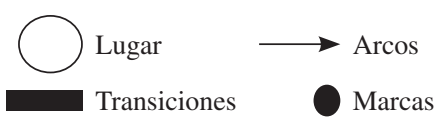

Figura 7. Elementos de una RdP.

Según [30], la fortaleza del modelado de las RdP radica en sus propiedades, que se dividen en dos grandes áreas, las dependientes del marcado inicial llamadas propiedades dinámicas o del comportamiento (alcanzabilidad, limitable o acotada, vivacidad, reversibilidad y estado inicial, cobertura, persistencia, distancia sincrónica) y las propiedades independientes del marcado, llamadas estructurales o estáticas (conservabilidad, limitación o acotado estructural, vivacidad estructural, repetibilidad, controlabilidad, consistencia).

Para la construcción de la RdP a partir del proceso, se comenzará con la traducción de las acciones, pasos, tareas, etapas o fases de este, a lugares y transiciones de la red. Como se muestra a continuación en la Tabla 2.

Tabla 2. Interpretaciones de una RdP.

\begin{tabular}{|l|l|l|}
\hline Lugares de entrada & Transiciones & Lugares de salida \\
\hline Precondiciones & Eventos & Post-condiciones \\
\hline Datos de entrada & Paso de cómputo & Datos de salida \\
\hline Necesidad de recursos & Acciones o tarea & Recursos liberados \\
\hline Condiciones & Cláusula lógica & Conclusiones \\
\hline
\end{tabular}


La construcción del grafo se realizará utilizando la simbología (Figura 7) siguiente:

\section{Cálculo de la ecuación de estados}

Luego del diseño de la red se procede a determinar las técnicas para su análisis, dentro de ellas las ecuaciones de estados conllevan al resultado final del proceso, en la bibliografía consultada se expone la ecuación de estados de una RdP como una ecuación matricial que define el estado de la red, dado un marcado inicial y una secuencia de disparos de transiciones habilitadas. La ecuación fundamental de una RdP (1) es:

$$
M d=M o+A^{T} \sum_{K=1}^{d} U_{k}
$$

Donde:

$M_{d}$ : es un vector columna de $m \times 1$, el vector de control o vector de disparo llamado.

$U_{k}$ : es un vector también columna de $n \times 1$.

$A^{T}$ : matriz $A$ es llamada de incidencia por que denota como cambiará el marcado, matriz de incidencia: $A=a i j$ donde $a i j=a i j+-a i j-$ donde estos son los pesos de los arcos de las transiciones a los lugares.

\section{Grafos de cobertura y de alcanzabilidad}

Dada la $\operatorname{RdP}\left(N, M_{0}\right)$ con marcado inicial $M_{0}$, se pueden obtener tantos nuevos marcados como transiciones habilitadas disparadas. Este proceso resulta en un grafo de marcados infinito para una RdP no acotada ${ }^{8}$. Para redes acotadas, el grafo de cobertura es llamado grafo de alcanzabilidad.

\section{Validación del procedimiento por medio de las propiedades de la $\mathbf{R d P}^{\mathbf{9}}$}

Obtenidas las técnicas de análisis, se procede a la evaluación del futuro comportamiento, para su validación.

Propiedades dinámicas o de comportamiento: Alcanzabilidad: principal propiedad dinámica cuyo objetivo es determinar si determinados estados son alcanzables o no. Cada disparo de una transición

\footnotetext{
8 Se explica más adelante.

9 Solo se tratarán las propiedades dinámicas por ser el objetivo la producción final y por ser las redes no ordinarias (sus arcos no siempre tienen peso uno)
}

habilitada modifica la distribución de los marcados dentro de la red, de acuerdo con las reglas de disparo. Esta propiedad es fácil de chequear si se dispone del grafo de alcanzabilidad y muy difícil, en el caso general, cuando no se dispone de él.

Limitable o acotada: se dice que la RdP está $k$-limitada si para todo marcado alcanzable se tiene que ningún lugar tiene un número de marcas mayor que $k$.

Vivacidad: se dice viva si en toda secuencia de ejecución infinita toda transición se puede disparar siempre, en el futuro (infinitas veces). Si las ejecuciones son finitas, por la naturaleza del sistema, no tiene sentido hablar de vivacidad, salvo que se haga una transformación del modelo. Si todas las transiciones de una red son vivas, la RdP se llama viva y así la red nunca se bloquea.

Reversibilidad y estado inicial: es reversible si para cualquier marcado alcanzable es posible volver al marcado inicial.

Cobertura: un marcado $M$ dentro de una RdP $\left(N, M_{0}\right)$ en un conjunto de marcados cubiertos o contenido, si existe un marcado $M$ ' dentro de $R$ $\left(N, M_{0}\right)$ tal que $M^{\prime}(p) \geq M(p)$ para cada lugar $p$ dentro de la red.

Persistencia: es persistente si para cualquiera de dos transiciones habilitadas, el disparo de una transición no deshabilitará a la otra transición.

Distancia sincrónica: grado de dependencia mutua entre dos eventos en un sistema condición/evento. Las redes de Petri como herramienta de modelación de procesos permiten una clara representación del flujo productivo directo e inverso de forma concurrente y efectiva.

\section{RESULTADOS}

La Empresa de Productos Lácteos de Holguín fue creada en 1981, presentándose como su actividad fundamental: la producción y distribución de las producciones lácteas, así como el acopio de leche fresca del sector estatal y privado además de otras actividades de servicios, para dar cumplimiento a su misión social. Cuenta con un total de ocho dependencias, siendo estas: el Combinado Lácteo Rafael Freyre que tiene como marco territorial la provincia (objeto de estudio en la investigación) y posee cuatro establecimientos distribuidos en los municipios de Moa, Mayarí y dos en Banes y un depósito en cada municipio antes mencionado y la dirección de la empresa en el municipio Holguín. 
El objetivo para que fuera creada la organización es la producción y distribución de leche para los niños 0-7 años y de yogurt para los niños de 7-13 años. Es una empresa provincial, que pertenece al sector de la industria alimenticia (MINAL) con subordinación nacional.

Misión: acopiar, producir y distribuir leche y sus derivados, productos a partir del frijol de soya mezclado físicos de alta calidad, a la canasta básica, el consumo social y el mercado captador de divisas. Visión: Ser un establecimiento eficiente, de reconocido prestigio nacional e internacionalmente, productor y comercializador de productos lácteos y derivados de la soya de alta calidad. Desarrollar nuevos productos para diversos mercados y exigencia con una distribución rápida, eficiente, segura y estable. Contar con un sistema de atención al hombre altamente motivado, que proporcione satisfacción, seguridad y estabilidad para el éxito.

Los cuadros, especialistas, técnicos y trabajadores están calificados y preparados en la gerencia moderna y están aptos para asimilar nuevas tecnologías.

Dentro de sus principales producciones se encuentra: Leche pasteurizada, yogurt natural y sabores para ambas monedas, mantequilla para ambas monedas, soyurt, leche de soya saborizada, helado para ambas monedas, queso crema para la venta en divisa.

El proceso en cuestión está enmarcado dentro de la línea de producción de helado y se rige por la norma NEIAL: $1611-59^{10}$. Este se realiza según lo expuesto en la Figura 8, donde se representa el flujo productivo mediante la técnica del diagrama de OTIDA (Operación-círculo, Transporte-flecha, Inspección-cuadrado, Demora-D y Almacenamientotriángulo), así como sus capacidades, que se inicia con la elaboración de la mezcla saborizada (que constituye el contenido de trabajo del puesto a analizar) para inmediatamente continuar en el puesto siguiente con la pasteurización y homogenización de la misma. Pasando luego a los tanques de maduración de los que se obtiene una mezcla final que se dirige al área de envase, aquí se inspecciona la calidad del producto el cual se destina un por ciento del producto

${ }^{10}$ Norma de calidad para la elaboración de productos derivados de la Leche.

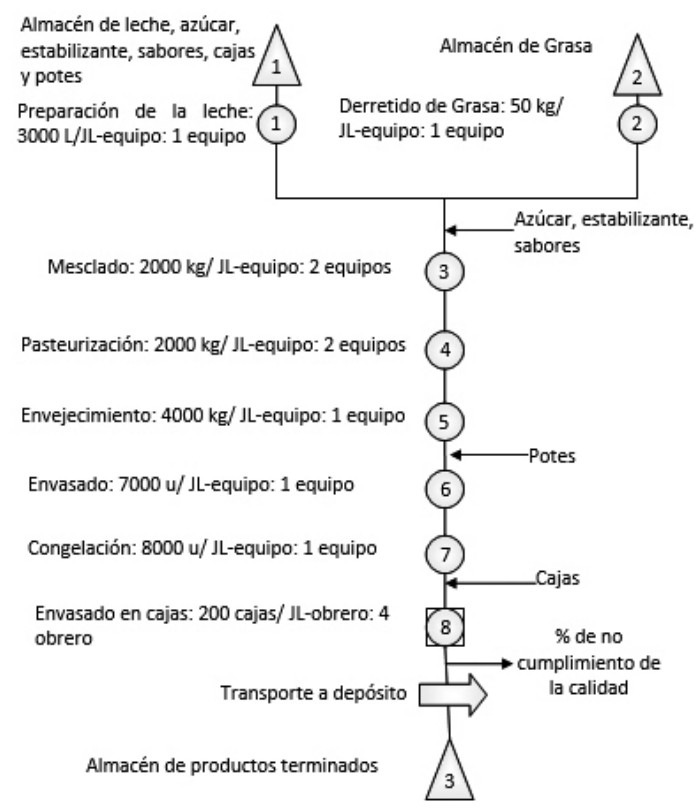

Figura 8. Descripción del proceso de producción de helados.

para obras de caridad, esos con menos calidad que los productos de comercialización.

Ya envasado el helado continúa hacia los almacenes, ya listo para comercializarse, concluyendo así el proceso de producción.

Como se puede observar en la Figura 9 se presenta la RdP que modela el proceso seleccionado. De igual forma en las Tablas 3, 4 y 5 se explica la leyenda de la RdP, atendiendo a los lugares, transiciones y arcos respectivamente donde se explica detalladamente la descripción del proceso y sus capacidades en cada instalación.

Luego se procedió a determinar la matriz de incidencia y matriz de disparos como se muestra en las Figuras 10 y 11.

Luego se procedió a calcular los marcados de la red por medio de la ecuación de estados, los resultados se muestran con el método del grafo de alcanzabilidad (Figura 12), teniendo en cuenta los resultados anteriores, del que se concluye la capacidad del proceso productivo, siendo el marcado nueve la producción vendida al cliente final con una actividad de 4.500 unidades diarias de helados. 


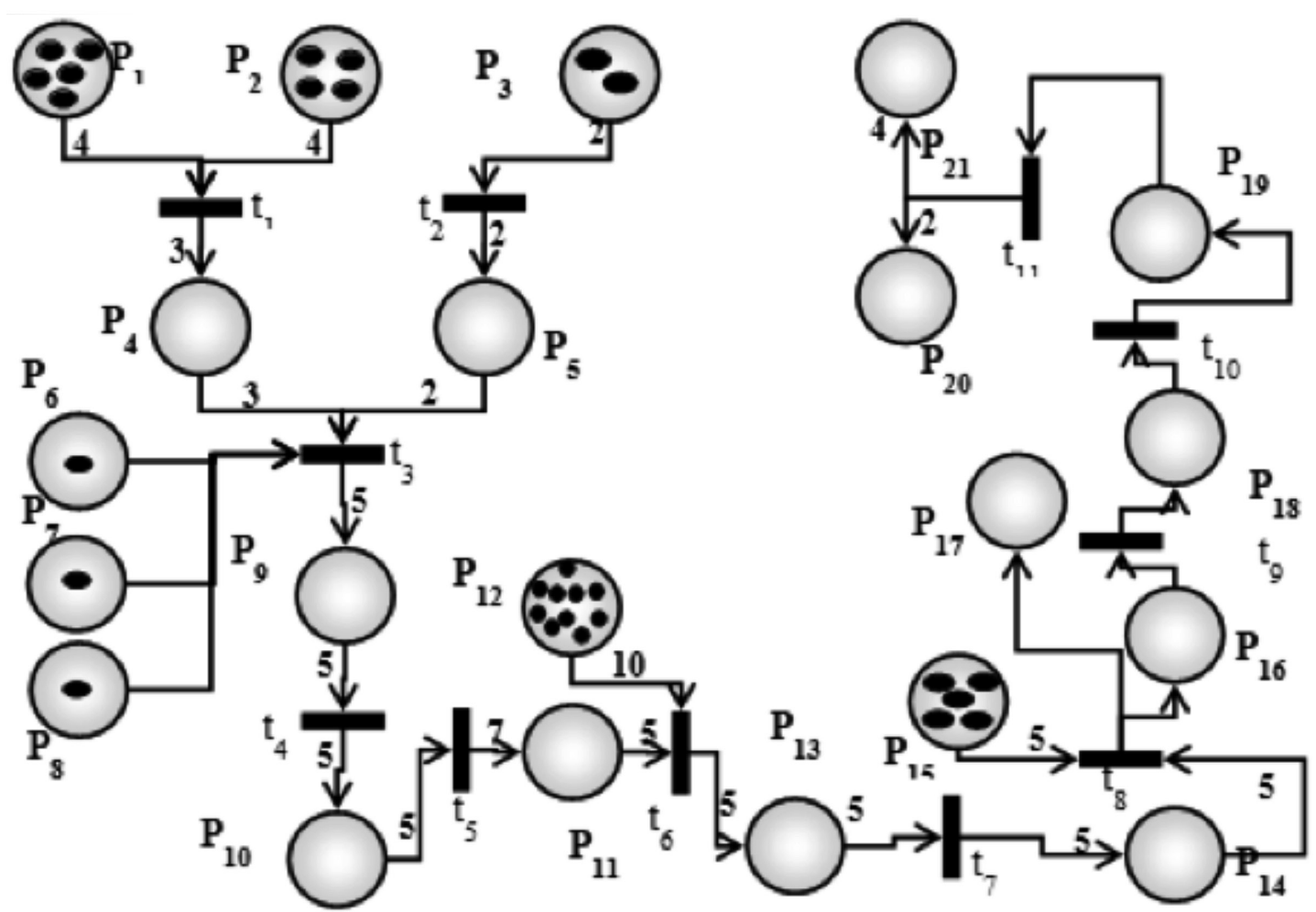

Figura 9. RdP del proceso seleccionado.

Tabla 3. Descripción de los lugares de la RdP.

\begin{tabular}{|c|l|}
\hline Lugares & \multicolumn{1}{|c|}{ Descripción : Representa la información asociada a la: } \\
\hline$P_{1}$ & Cantidad de litros de agua en el almacén 1: cincos tanques de 6.000 litros en una Jornada Laboral (JL) \\
\hline$P_{\mathbf{2}}$ & Cantidad de leche en polvo en el almacén 1: cuatros bultos sacos de $900 \mathrm{~kg}$ de leche en polvo \\
\hline $\mathrm{P}_{\mathbf{3}}$ & Cantidad de tanques de $20 \mathrm{~kg}$ de grasa en el almacén 2 \\
\hline $\mathrm{P}_{\mathbf{4}}$ & Cantidad de toneladas de leche derretida \\
\hline $\mathrm{P}_{\mathbf{5}}$ & Cantidad de tanques de grasa derretida \\
\hline $\mathrm{P}_{\mathbf{6}}$ & Cantidad de sabores concentrados extraída del almacén: 252 gramos \\
\hline $\mathrm{P}_{\mathbf{7}}$ & Cantidad de estabilizantes extraída del almacén: $100 \mathrm{~kg}$ \\
\hline $\mathrm{P}_{\mathbf{8}}$ & Cantidad de azúcar refino extraída del almacén: una tonelada \\
\hline $\mathrm{P}_{\mathbf{9}}$ & Cantidad de tanques de 700 kg de mezcla \\
\hline $\mathrm{P}_{\mathbf{1 0}}$ & Cantidad de tanques de 700 kg de pasta \\
\hline $\mathrm{P}_{\mathbf{1 1}}$ & Cantidad de tanques de 500 kg de pasta envejecida \\
\hline $\mathrm{P}_{\mathbf{1 2}}$ & Cantidad de bultos de potes de helado existente en el almacén: 500 potes por bultos \\
\hline $\mathrm{P}_{\mathbf{1 3}}$ & Cantidad de lotes de miles de unidades envasados \\
\hline $\mathrm{P}_{\mathbf{1 4}}$ & Cantidad de lotes de miles de unidades congelados \\
\hline $\mathrm{P}_{\mathbf{1 5}}$ & Cantidad de bultos de 500 cajas en el almacén \\
\hline $\mathrm{P}_{\mathbf{1 6}}$ & Cantidad de bultos de 500 cajas en el embalaje \\
\hline $\mathrm{P}_{\mathbf{1 7}}$ & Cantidad de bultos de 50 cajas unidades defectuosas \\
\hline $\mathrm{P}_{\mathbf{1 8}}$ & Cantidades de unidades en el depósito \\
\hline $\mathrm{P}_{\mathbf{1 9}}$ & Cantidad de unidades en lugar de ventas \\
\hline $\mathrm{P}_{\mathbf{2 0}}$ & Cantidad de bultos de 500 cajas almacenadas por el vendedor \\
\hline $\mathrm{P}_{\mathbf{2 1}}$ & Cantidad de bultos de 500 envases consumidos al momento \\
\hline
\end{tabular}


Tabla 4. Descripción de los arcos de la RdP.

\begin{tabular}{|c|c|}
\hline $\operatorname{Arcos}$ & Descripción \\
\hline $\mathrm{P}_{1^{-} \mathrm{t}_{1}}$ & Peso 4: representa la cantidad de tanques agua para derretir la leche \\
\hline $\mathrm{P}_{2}-\mathrm{t}_{1}$ & Peso 4: representa las cantidades de 0,9 toneladas de leche para derretir \\
\hline $\mathrm{P}_{3}-\mathrm{t}_{2}$ & Peso 2: representa la cantidad de tanques de $20 \mathrm{~kg}$ de grasa para derretir \\
\hline $\mathrm{t}_{1}-\mathrm{P}_{4}$ & Peso 3: representa la cantidad de toneladas de leche derretida \\
\hline $\mathrm{t}_{2}-\mathrm{P}_{5}$ & Peso 2: representa la cantidad de tanques de $20 \mathrm{~kg}$ de grasa derretida \\
\hline $\mathrm{P}_{4}-\mathrm{t}_{3}$ & Peso 3: representa la cantidad de toneladas de leche a mezclar \\
\hline $\mathrm{P}_{5}-\mathrm{t}_{3}$ & Peso 2: representa la cantidad de tanques de grasa a mezclar \\
\hline $\mathrm{P}_{6}-\mathrm{t}_{3}$ & Peso 1: representa la cantidad de concentrado necesario a mezclar \\
\hline $\mathrm{P}_{7}-\mathrm{t}_{3}$ & Peso 1: representa la cantidad de estabilizante necesario a mezclar \\
\hline $\mathrm{P}_{8}-\mathrm{t}_{3}$ & Peso 1: representa la cantidad de toneladas de azúcar refino necesaria a mezclar \\
\hline $\mathrm{t}_{3}-\mathrm{P}_{9}$ & Peso 5: cantidad de lotes de $700 \mathrm{~kg}$ de mezcla realizada \\
\hline $\mathrm{P}_{9}-\mathrm{t}_{4}$ & Peso 5: cantidad de lotes de $700 \mathrm{~kg}$ de mezcla a pasteurizar \\
\hline$t_{4}-P_{10}$ & Peso 5: cantidad de lotes de $700 \mathrm{~kg}$ de pasta realizada \\
\hline $\mathrm{P}_{10} \mathrm{-t}_{5}$ & Peso 5: cantidad de lotes de $700 \mathrm{~kg}$ de pasta a envejecer \\
\hline $\mathrm{t}_{5}-\mathrm{P}_{11}$ & Peso 7: cantidad de lotes de $500 \mathrm{~kg}$ de pasta envejecida \\
\hline $\mathrm{P}_{11^{-} \mathrm{t}_{6}}$ & Peso 5: cantidad de lotes de $500 \mathrm{~kg}$ a envasar \\
\hline $\mathrm{P}_{12} \mathrm{-t}_{6}$ & Peso 10 cantidad de bultos de 500 envases de $0,5 \mathrm{~kg} / \mathrm{u}$ para envasar \\
\hline $\mathrm{t}_{6}-\mathrm{P}_{13}$ & Peso 5: cantidad de lotes de miles de unidades envasadas \\
\hline $\mathrm{P}_{13^{-}} \mathrm{t}_{7}$ & Peso 5: cantidad de lotes de miles de unidades envasadas a conservar \\
\hline $\mathrm{t}_{7}-\mathrm{P}_{14}$ & Peso 5: cantidad de lotes de miles de unidades conservadas \\
\hline $\mathrm{P}_{14} \mathrm{-t}_{8}$ & Peso 5: cantidad de lotes de miles de unidades conservadas a envasar en cajas \\
\hline $\mathrm{P}_{15} \mathrm{-}_{8}$ & Peso 5: cantidad de centenas de cajas para envasar \\
\hline $\mathrm{t}_{\mathbf{8}}-\mathrm{P}_{16}$ & Peso 1: 450 cajas envasadas \\
\hline $\mathrm{t}_{\mathbf{8}}-\mathrm{P}_{17}$ & Peso 1: 50 cajas que no cumple con la calidad requerida \\
\hline $\mathrm{P}_{16} \mathrm{-t}_{9}$ & Peso 1: cantidad de cajas a depositar (todas) \\
\hline $\mathrm{t}_{\mathbf{9}}-\mathrm{P}_{\mathbf{1 8}}$ & Peso 1: cantidad de cajas depositadas (todas) \\
\hline $\mathrm{P}_{18} \mathrm{t}_{10}$ & Peso 1: cantidad de cajas necesarias para vender (todas) \\
\hline $\mathrm{t}_{10}-\mathrm{P}_{19}$ & Peso 1: cantidad de cajas vendidas (todas) \\
\hline $\mathrm{P}_{19} \mathrm{t}_{11}$ & Peso 1: cantidad de cajas necesarias para el consumo (todas) \\
\hline $\mathrm{t}_{11} \mathrm{P}_{20}$ & Peso 1: cantidad de cajas almacenadas por el vendedor \\
\hline $\mathrm{t}_{11}-\mathrm{P}_{21}$ & Peso 1: cantidad de lotes de miles de unidades consumidas al momento por el cliente \\
\hline
\end{tabular}

Tabla 5. Descripción de las transiciones de la RdP.

\begin{tabular}{|c|l|}
\hline Transiciones & \multicolumn{1}{c|}{ Descripción } \\
\hline$t_{1}$ & Derretido de leche en polvo: 2 horas \\
\hline$t_{2}$ & Derretido de grasa: 30 minutos \\
\hline$t_{3}$ & Mezclado de la materia prima: 2 horas \\
\hline$t_{4}$ & Pasteurización de la mezcla: 2 horas \\
\hline$t_{5}$ & Envejecimiento de la pasta: 2 horas \\
\hline$t_{6}$ & Envasado en potes: 2 horas \\
\hline$t_{7}$ & Congelación del producto: 2 horas \\
\hline$t_{8}$ & Envasado en caja (10 u/caja): 1 hora \\
\hline$t_{9}$ & Transporte del producto terminado: 30 minutos \\
\hline$t_{10}$ & Venta del producto: 4 horas \\
\hline$t_{11}$ & Consumo del producto al momento: 4 horas \\
\hline
\end{tabular}




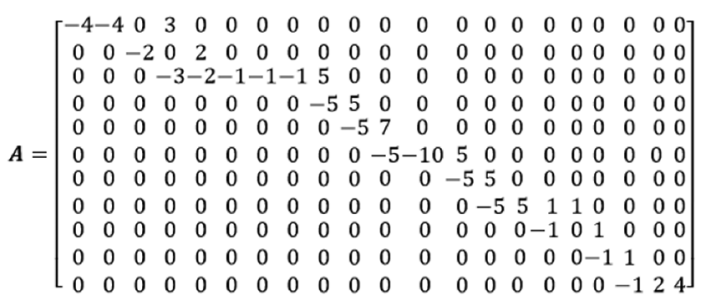

Figura 10. Matriz de incidencia $(A)$ de la red.

$$
U_{k}=\left[\begin{array}{llllllllll}
1 & 0 & 0 & 0 & 0 & 0 & 0 & 0 & 0 & 0 \\
1 & 0 & 0 & 0 & 0 & 0 & 0 & 0 & 0 & 0 \\
0 & 1 & 0 & 0 & 0 & 0 & 0 & 0 & 0 & 0 \\
0 & 0 & 1 & 0 & 0 & 0 & 0 & 0 & 0 & 0 \\
0 & 0 & 0 & 1 & 0 & 0 & 0 & 0 & 0 & 0 \\
0 & 0 & 0 & 0 & 1 & 0 & 0 & 0 & 0 & 0 \\
0 & 0 & 0 & 0 & 0 & 1 & 0 & 0 & 0 & 0 \\
0 & 0 & 0 & 0 & 0 & 0 & 1 & 0 & 0 & 0 \\
0 & 0 & 0 & 0 & 0 & 0 & 0 & 1 & 0 & 0 \\
0 & 0 & 0 & 0 & 0 & 0 & 0 & 0 & 1 & 0 \\
0 & 0 & 0 & 0 & 0 & 0 & 0 & 0 & 0 & 1
\end{array}\right]
$$

Figura 11. Matriz de disparos $(U)$ de la red.

El marcado final representado en la red de la Figura 13, da información relacionada a 1.000 cajas almacenadas por el vendedor como promedio diariamente, en aceptable estados de disponibilidad, igualmente 2.000 envases plásticos, el resto de los envases y embalajes no se pueden reutilizar, por deficiente disponibilidad o por no ser consumidos al momento. También se despilfarra un tanque de

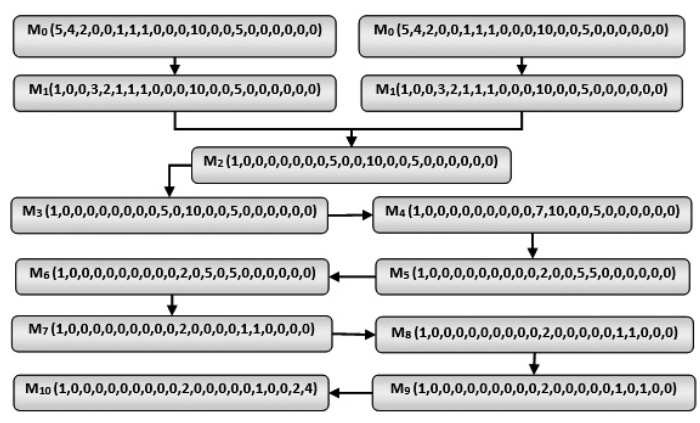

Figura 12. Grafo de alcanzabilidad.

agua que puede ser utilizado en el lavado de las áreas, o simplemente ahorrarla.

\section{Validación del proceso}

Alcanzabilidad: cada disparo modifica la distribución de los marcados en la RdP, de acuerdo con la reglas de disparos. Todos los marcados son alcanzables, el marcado final $M_{10}$ es alcanzable desde $M_{0}$ según la secuencia de los disparos (Figura 12) en el proceso seleccionado.

Limitable o acotada: la RdP no es acotada según la red de las Figuras 9 y 13, por cada disparo se obtiene una $M$ cantidad de disparos menor o igual que la anterior, según la RdP el número de marcado es diferente al peso de su arco de salida $\left(\mathrm{P}_{\mathbf{1}}, \mathrm{P}_{\mathbf{1 1}}\right)$, por lo que es de razón diferente a uno y se puede

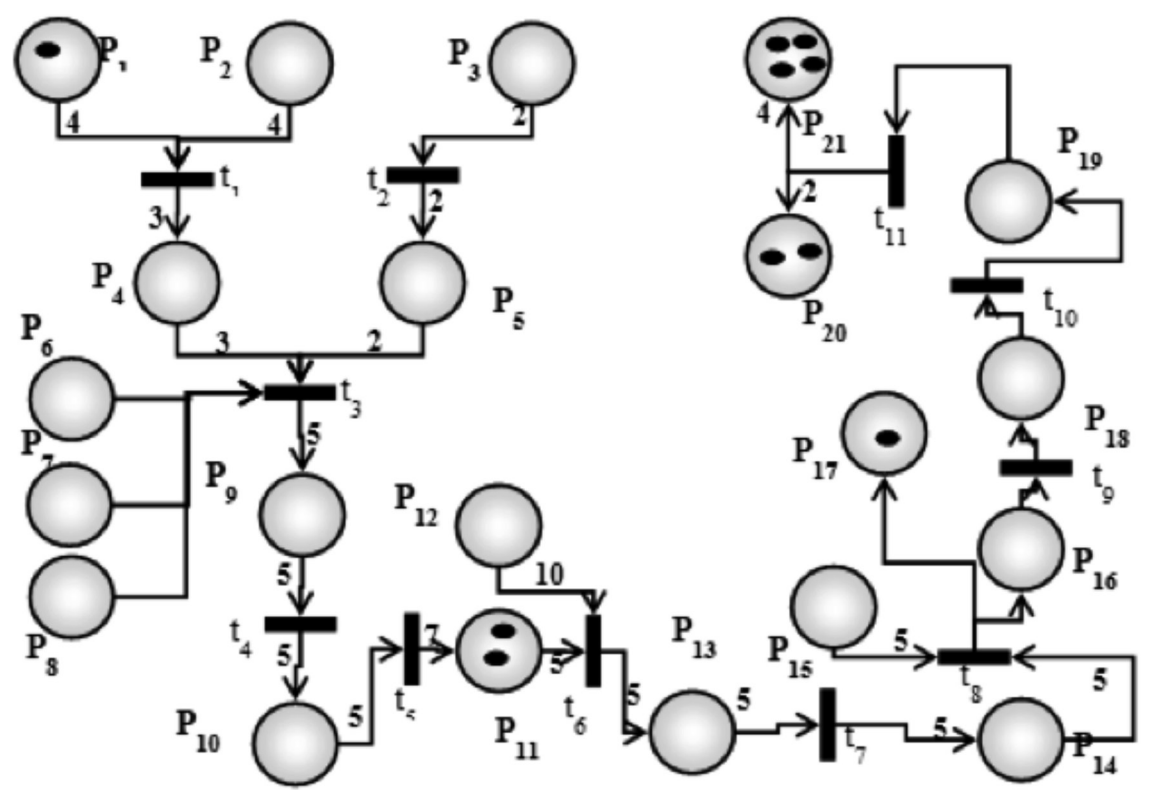

Figura 13. Marcado final de la RdP. 
decir que no es limitada a uno por lo que la RdP no es segura y no será efectiva.

Vivacidad: la RdP no es viva, pues una vez alcanzado el estado final por las propias características del proceso en un día no será necesaria una nueva secuencia de disparos, pues es la producción máxima diaria, esta propiedad no es de interés en un día de producción, fuera de este período, se tendrán en cuenta las ventas que me delimitan los suministros, volviendo así, a ejecutarse el proceso.

Reversibilidad y estado inicial: no es reversible pues una vez alcanzado los diferentes objetivos del proceso, no será necesario modificar las condiciones iniciales y volver al estado inicial en un día.

Cobertura: dado que el procedimiento seleccionado no es cíclico, el grafo de cobertura coincide con el grafo de alcanzabilidad por lo que se puede asegurar que el marcado final se alcanza la venta de los helados. Persistencia: a lo largo de la RdP, el disparo de una transición no deshabilitará a otra pues las transiciones no comparten ningún recurso.

Distancia sincrónica: partiendo de que la RdP es un sistema inherentemente paralelo y (o) concurrente, dos transiciones que estén capacitadas y no interactúan entre sí, que pueden disparar de forma independiente. En esta RdP no es necesario "sincronizar" estos resultados ya que no es un requerimiento del proceso seleccionado pues aquellas que pueden ser disparadas al mismo tiempo, no impedirán su alcanzabilidad.

Se comprobó que este logra su objetivo pero no de forma segura, por lo que no es efectiva ya que no garantiza que nunca entrará en un estado no válido, o sea sigue instrucciones que no se deben realizar, es persistente, etc. y en cuanto a las propiedades que no se cumplen, se considera que no son necesarias para este tipo de proceso. Se pudo comprobar que el modelo supone una aproximación adecuada de la realidad para los objetivos particulares del proceso, los datos generados de la simulación del modelo, reproducen de forma adecuada el comportamiento del sistema real con la comprobación del comportamiento del modelo con las propiedades dinámicas.

\section{Propuesta mejorada}

Para la mejora continua e incremento de la productividad se establecieron puntos de clasificadores de los envases y embalajes, que fueron clasificados como envases retornables ${ }^{11}$ : así como puntos de lavados de envases y reparaciones de cajas, modificando el proceso según la Figura 14,

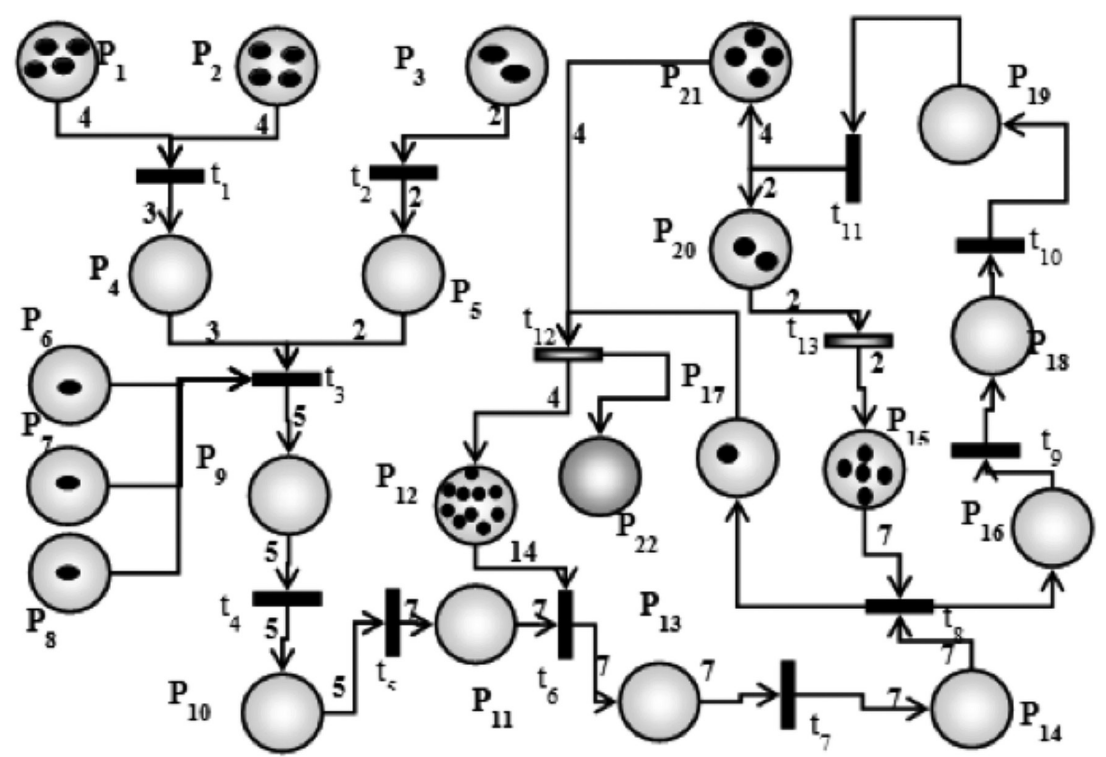

Figura 14. RdP del proceso propuesto.

\footnotetext{
11 Recipientes destinados a la venta con el producto que contienen y que es reusado por la empresa productora.
} 
incrementándose nuevos elementos como los establecidos en la Tabla 6.

Tabla 6. Descripción de los nuevos elementos de la RdP.

\begin{tabular}{|l|l|}
\hline \multicolumn{1}{|c|}{$\mathbf{P}_{\mathbf{2 2}}$} & \multicolumn{1}{|c|}{ Cantidad de envases a reciclar } \\
\hline $\mathrm{t}_{\mathbf{1 2}}$ & $\begin{array}{l}\text { Operación de clasificación y lavados de } \\
\text { envases plásticos }\end{array}$ \\
\hline $\mathrm{t}_{\mathbf{1 3}}$ & $\begin{array}{l}\text { Operación de transportación de cajas } \\
\text { recuperadas }\end{array}$ \\
\hline $\mathrm{P}_{\mathbf{2 1}}-\mathrm{t}_{\mathbf{1 2}}$ & $\begin{array}{l}\text { Peso 4: Cantidad de bultos de 500 } \\
\text { envases plásticos para clasificar }\end{array}$ \\
\hline $\mathrm{P}_{\mathbf{1 7}}-\mathrm{t}_{\mathbf{1 2}}$ & $\begin{array}{l}\text { Peso 1: Cantidad de envases plásticos } \\
\text { para clasificar }\end{array}$ \\
\hline $\mathrm{P}_{\mathbf{2 0}}-\mathrm{t}_{\mathbf{1 3}}$ & $\begin{array}{l}\text { Peso 2: Cantidad de centenas de cajas } \\
\text { para transportar }\end{array}$ \\
\hline $\mathrm{t}_{\mathbf{1 2}}-\mathrm{P}_{\mathbf{1 2}}$ & $\begin{array}{l}\text { Peso 5: Cantidad de bultos de 500 } \\
\text { envases recuperados para el embalaje }\end{array}$ \\
\hline $\mathrm{t}_{\mathbf{1 3}}-\mathrm{P}_{\mathbf{1 5}}$ & $\begin{array}{l}\text { Peso 2: Cantidad de centenas de cajas } \\
\text { para el embalaje }\end{array}$ \\
\hline
\end{tabular}

Luego se procedió a calcular los marcados mediante las ecuaciones de estados obteniendose los resultados expuestos en la Figura 15.

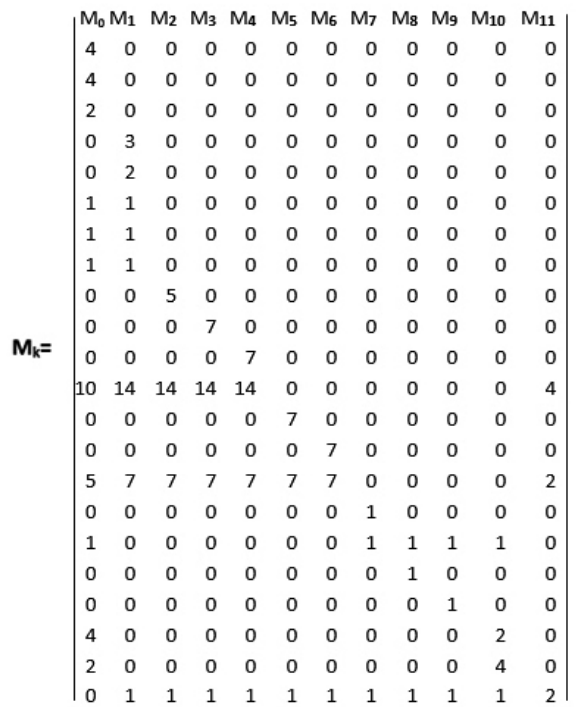

Figura 15. Matriz de marcados $(M)$ de la red.

El objetivo final fue logrado nuevamente, obteniéndose según el marcado $\mathrm{M}_{9}$ representado en la Figura 16, lográndose un nivel de producción de 7.000 unidades diarias, y en el marcado final (Figura 15) se puede apreciar 2.000 envases plásticos para el vertedero y 2.000 envases y 1.000 cajas esperando para ser reutilizados.

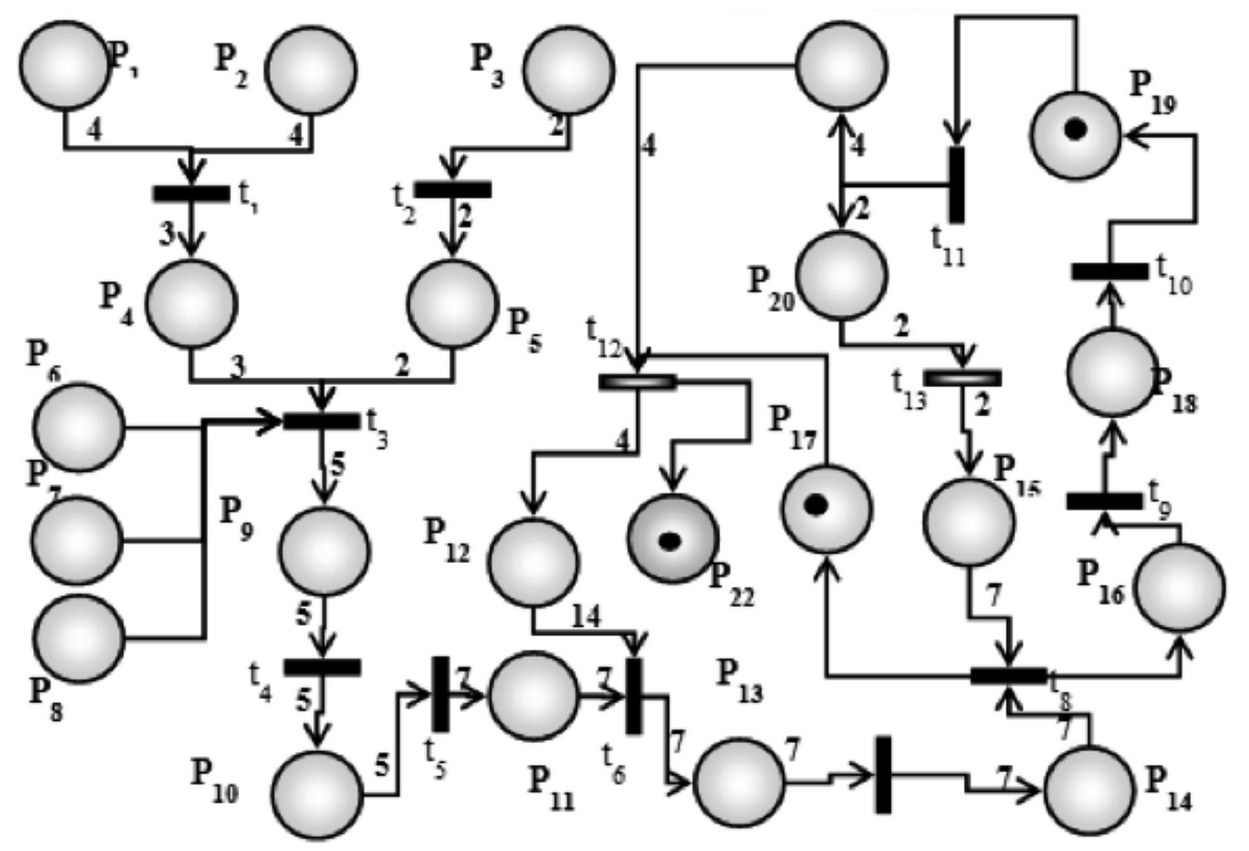

Figura 16. Marcado $\mathrm{M}_{9}$ de la RdP. 
Se comprobó que este logra su objetivo de forma segura, por lo que es efectivo ya que garantiza que nunca entrará en un estado no válido, o sea sigue instrucciones que se deben realizar, es persistente, etc. Se pudo comprobar que el modelo supone una aproximación adecuada de la realidad para los objetivos particulares del proceso, concluyéndose valido, constituyendo un salto en la mejora continua en cuanto a incremento de la producción y efectividad.

\section{DISCUSIÓN}

Se diseñó el proceso de logística inversa con la herramienta seleccionada (en la simulación realizada el centro de retornos recibirá y procesará 2.000 envases y 1.000 cajas), que permitirán en base a la información suministrada por la empresa respecto a tiempos, costos y personal el análisis de los resultados para los parámetros incremento de la productividad $\mathrm{y}$ eficiencia de las operaciones.

En la Tabla 7 se muestran los resultados obtenidos para los dos procesos de logística inversa (actual y propuestos) modelados mediante redes de Petri.

Ante situaciones tan diferentes se ha comprobado que la mejora se produce en los procesos directamente relacionados con la logística inversa en caso
Tabla 7. Comparación de la propuesta.

\begin{tabular}{|l|r|r|}
\hline \multicolumn{1}{|c|}{ Criterio } & \multicolumn{1}{c|}{ Actual } & Propuesta \\
\hline Producción & $4500 \mathrm{u}$ & $6500 \mathrm{u}$ \\
\hline Ingresos & $\$ 112500,00$ & $\$ 162500,00$ \\
\hline Costos & $\$ 98725,96$ & $\$ 99625,26$ \\
\hline Utilidad & $30 \mathrm{t}$ & $32 \mathrm{t}$ \\
\hline $\begin{array}{l}\text { Trabajadores directos a la } \\
\text { producción }\end{array}$ & $150 \mathrm{u} / \mathrm{t}$ & $203,25 \mathrm{u} / \mathrm{t}$ \\
\hline Productividad & no & $\mathrm{si}$ \\
\hline Efectividad & baja & alta \\
\hline Validez del proceso & & $\$ 62874,74$ \\
\hline
\end{tabular}

específico de la reutilización de los envases, cumpliendo un objetivo prioritario de la empresa "la satisfacción del cliente", al aumentar los niveles de ventas del producto. Estos resultados han sugerido al grupo de trabajo a realizar un posterior estudio detallado de los procesos relacionados con el envase y embalaje, dentro del centro de clasificación con el fin de plantear nuevas mejoras.

Tras la comparación de ambas alternativas se puede concluir que la propuesta ofrece más posibilidades que la actual, su comportamiento es más efectivo, aumento de las utilidades y un incremento de la productividad de un $35,5 \%$ (cociente entre la

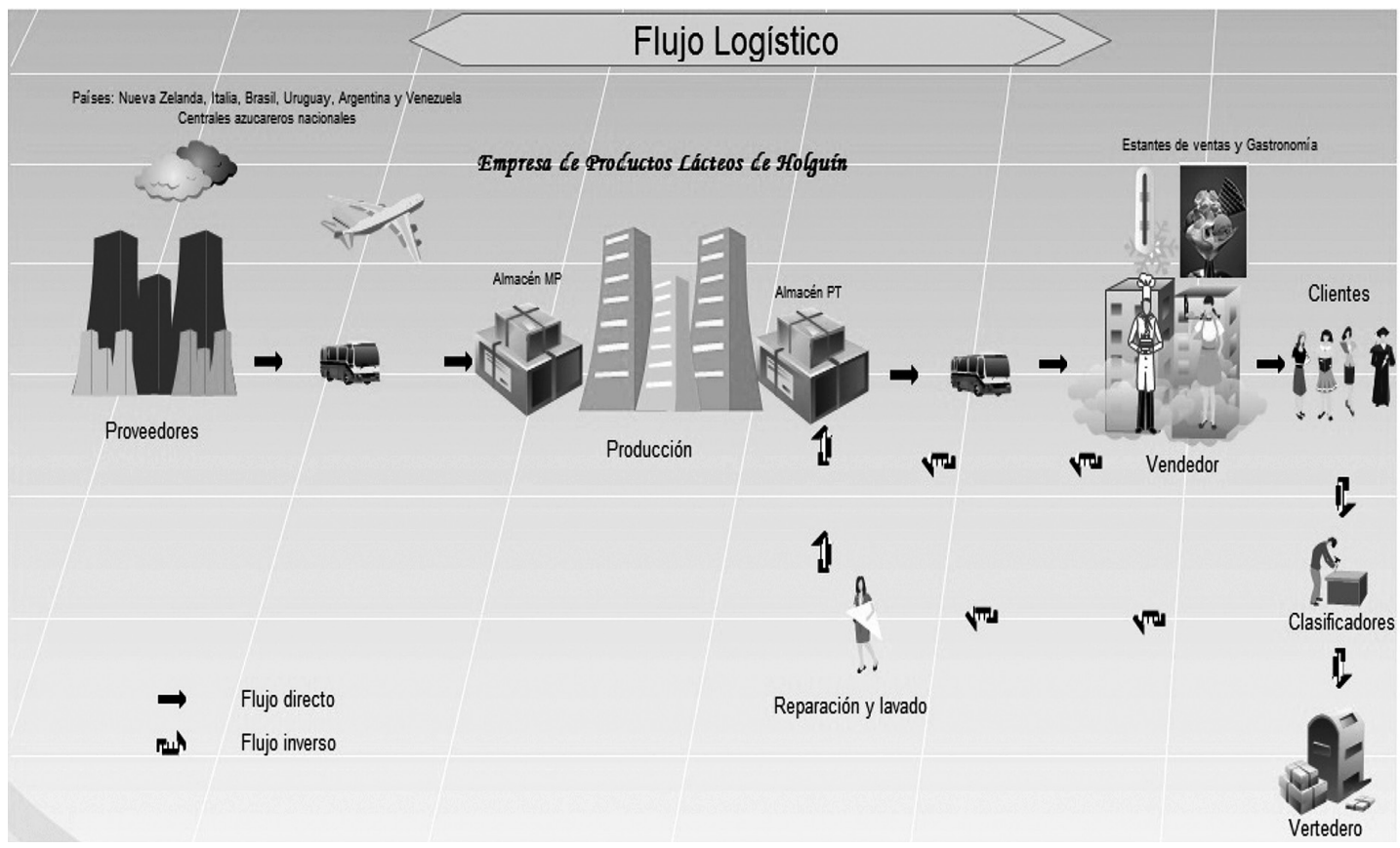

Figura 17. Flujo logístico del proceso propuesto. 
variación de la productividad y la productividad inicial), por lo que se presenta como una propuesta económica para la producción de helados. El flujo logístico queda como se representa en la Figura 17.

\section{CONCLUSIONES}

Se demostró la necesidad de un enfoque para la modelación de los procesos de logística inversa, mediante la descripción del flujo de producción directo. El artículo permite ratificar como las redes de Petri son una herramienta muy útil en el modelado de procesos productivos, debido a que soportan una representación gráfica que facilita la comprensión del sistema modelado y simultáneamente, posibilitan un análisis formal en el establecimiento, verificación y validación prospectiva del proceso por medio de su comportamiento, posibilitando implantar la logística inversa mediante la reutilización, lo que contribuyó al incremento de los beneficios de la organización como ganancias, productividad y efectividad del proceso.

\section{RECOMENDACIONES}

1. Profundizar en los subprocesos de producción respecto a la valides de estos para incrementar sus capacidades.

2. Indagar en otras alternativas de la logística inversa para la mejora de la imagen de la empresa.

3. Extender el desarrollo científico tecnológico al medio industrial de tal forma que la utilización de modelos simulados con redes de Petri permita mejorar los sistemas de manufactura reales, $\mathrm{y}$ elimine desbalances de producción, bloqueos, subutilización de sistemas, etc.

4. Establecer procedimientos de verificación y validación para los modelos de simulación.

\section{REFERENCIAS}

[1] A.J. Acevedo Urquiaga. "Modelo de Gestión Colaborativa del Flujo Logístico". Tesis en opción al título de Doctor en Ciencias Técnicas. Instituto Superior Politécnico "José Antonio Echeverría". 2013. URL: http://catedragc.mes.edu.cu/repositorios/

[2] M. Feitó Cespón, R. Cespón Castro y M. Alejandro Rubio Rodríguez. "Modelos de optimización para el diseño sostenible de cadenas de suministros de reciclaje de múltiples productos". Ingeniare. Rev. chil. ing. Vol. 24 $\mathrm{N}^{\circ}$ 1, pp. 135-148. 2016. URL: http://www. ingeniare.cl/index.php?option=com_ingeni are $\&$ view $=$ va\& aid $=474 \&$ vid $=86 \&$ lang $=e s$

[3] M.C. Vélez Gallego, D. Avalencia Ramírez and C.A. Castro Zuluaga. "A simulationoptimization heuristic for configuring a selective pallet rack system". Ingeniare. Rev. chil. ing. Vol. $20 \mathrm{~N}^{\mathrm{o}}$ 1, pp. 17-24, 2012. ISSN: 0718-3305. http://dx.doi.org/10.4067/ S0718-33052012000100003.

[4] J.O. Hernández, S. Hernández y I. Flores. "Algoritmo recocido simulado para el problema de la programación del tamaño del lote económico bajo el enfoque de ciclo básico". Ingeniare. Rev. chil. ing. Vol. 19 No 3 , pp. 473-485. 2011. ISSN: 0718-3305. URL: http://dx.doi.org/10.4067/S0718-33052011 000300015.

[5] R.T. Araújo, M.E. Araújo, F.N. Medeiros y G.C. Barroso. "Modelagem de um sistema de gestãon a Educação a Distância no Brasil utilizando redes de Petri Coloridas". Ingeniare. Revista chilena de ingeniería. Vol. $23 \mathrm{~N}^{\circ}$ 1. 2015. http:// www.scielo.cl/scielo.php?pid=S071833052015000100016\&script=sci_arttext

[6] M.M.H. Cely. "Modelado e implementación de un sistema automático de detección y diagnóstico de fallas basado en redes de Petri para el proceso hogar en la generación de vapor". Scientia et Technica. Vol. $18 \mathrm{~N}^{\circ} 4$, pp. 599-605. 2013. URL: http://www.redalyc. org/html/849/84929984003/

[7] H. Gutiérrez y A.G. Muñoz. "Generación de diagramas ladder mediante el uso de redes de Petri difusas". Revista Vínculos. Vol. 10 № 2, pp. 367-280. 2013. URL: http://revistas. udistrital.edu.co/ojs/index.php/vinculos/ article/view/6523

[8] M. Mozada y J.M. Velasco. "Modelado dinámico basado en redes de Petri para el modelo de integración empresarial Actor de Empresa". Scientia et Technica. Vol. $1 \mathrm{~N}^{\circ}$ 44, pp. 140145. 2010. URL: http://revistas.utp.edu.co/ index.php/revistaciencia/article/view/1797

[9] L.D. Murillo Soto. "Simulación de un sistema de manufactura flexible con redes de Petri coloreadas". Tecnología en Marcha. Vol. 23 $\mathrm{N}^{\mathrm{o}}$ 1, pp. 47. 2011. URL: http://revistas.tec. ac.cr/index.php/tec_marcha/article/view/133 
[10] M. Narciso, M. Piera y J. Figueras. "Optimización de sistemas logísticos mediante simulación: Una metodologia basada en redes de Petri coloreadas". RIAII. Vol. $2 \mathrm{~N}^{\circ}$ 4, pp. 54-65. 2010. URL: http://upcommons.upc.edu/bitstream/ handle/2117/17423/metodologia.pdf

[11] A. Morales Varela, J.A. Rojas Ramírez, L.H. Hernández Gómez, Á. Morales González y M.Y. Jiménez Reyes. "Modelo de un sistema de producción esbelto con redes de Petri para apoyar la toma de decisiones". Ingeniare. Revista chilena de ingeniería. Vol. $23 \mathrm{~N}^{\circ} 2$, pp. 182-195. 2015. URL: http://www.scielo. cl/pdf/ingeniare/v23n2/art04

[12] O.A. Rojas Alvarado. "Modelado dinámico del proceso de trazabilidad de producto con redes de Petri para WorkFlow (WF-Net)". Épsilon. Vol. I No 19, pp. 117-144. 2012. URL: http://revistas.lasalle.edu.co/index. php/ep/article/view/2248

[13] S. Sánchez, A. Herrera y R.C.A. Rovetto. "Análisis y modelamiento del Canal de Panamá a través de las redes de Petri". En: Proceedings of the 7th Euro American Conference on Telematics and Information Systems. 2014. URL: http://dl.acm.org/ citation.cfm?id=2590667

[14] M.E. Villapol. "Análisis de las propiedades del establecimiento de la conexión Bluetooth Bandabase usando Redes de Petri Coloreadas". Computación y Sistemas. Vol. $16 \mathrm{~N}^{\mathrm{o}} 4$, pp. 433-446. 2012. URL: http://www. repositoriodigital.ipn.mx/handle/1234567 89/14686? show=full

[15] G. Zapata, B. Hoyos y L. Quintero. "Diseño del sistema automático para una planta piloto de recubrimientos electrolíticos. Parte I: Modelo mediante Redes de Petri Jerárquicas". Revista Facultad de Ingeniería. Vol. $1 \mathrm{~N}^{\circ}$ 45, pp. 67-76. 2008. URL: http:// www.scielo.org.co/scielo.php?pid=S0120$62302008000300006 \&$ script=sci_arttext

[16] L.O. Vega de la Cruz y A.F. Nieves Julbe. "Validación prospectiva de modelos académicos".Enl@ce. Vol. 12 №3, pp.71-98. 2015. ISSN: 1690-7515. URL: http:// produccioncientificaluz.org/index.php/ enlace/article/view/20628

[17] Y. Pardillo Bá ez. "Modelo de Diseño de Nodos de Integración en las Cadenas de Suministro".
Tesis en opción al título de Doctor en Ciencias Técnicas. Instituto Superior Politécnico José Antonio Echeverría. 2013. URL: http:// catedragc.mes.edu.cu/repositorios/

[18] V. Reyes, D. Zavala y J. Gálvez. "Una revisión del proceso de la logística inversa y su relación con la logística verde". 2008. URL: www. dialnet.unirioja.es/descarga/ 3997991.pdf

[19] K.J. Aguilar Vargas, J.A. Manrique y L.L. Salamanca Martínez. "Propuesta de planificación, desarrollo y control a los procesos de la logística inversa en la empresa Une Telecomunicaciones S.A.”. Universidad Militar Nueva Granda. Bogotá, Colombia. 2013. URL: URL: repository.unimilitar.edu. co/handle/10654/7061

[20] J.A. Knudsen González. "Diseño y gestión de la cadena de suministro de los residuos agroindustriales de la caña de azúcar. Aplicación a los residuos agrícolas cañeros, el bagazo y las mieles". Tesis para optar al grado de Doctor. Universidad Central "Marta Abreu" de Las Villas, 2005. URL: http:// catedragc.mes.edu.cu/repositorios/

[21] X. Llauró Fábregas. "Estudio de la aplicación de sistemas basados en el conocimiento a la operación de una planta de tratamiento de residuos sólidos urbanos por valorización energética". Tesis para optar al grado de Doctor. Instituto Politécnico "José Antonio Echeverría”. La Habana, Cuba. 1999. http:// catedragc.mes.edu.cu/repositorios/

[22] J.C. Meneses Marcel. "Perfeccionamiento de las rutas de distribución de la Empresa Suchel Trans para la provincia de Sancti Spíritus, utilizando el método de Optimización por Colonia de Hormigas". Universidad Central "Marta Abreu" de Las Villas. 2009. URL:_http://catedragc.mes. edu.cu/repositorios/

[23] R. Martín. "El efecto látigo (bullwhip) en las cadenas de suministro y la dependencia de los agentes que las integran". Universidad Pontificia Comillas de Madrid: ETS de Ingeniería (ICAI). 2006. URL: http:// catedragc.mes.edu.cu/repositorios/

[24] S.A. Blanco Londono y T. Rodríguez Chaparro. "Producción de biohidrógeno a partir de residuos mediante fermentación oscura: una revisión crítica (1993-2011)". Ingeniare. Rev. chil. ing. Vol. $20 \mathrm{~N}^{\mathrm{o}}$ 3, pp. 398-411. 
2012. ISSN: 0718-3305. URL: http://dx.doi. org/10.4067/S0718-33052012000300014

[25] Y. Broche Fernández. "Modelo y procedimiento para la gestión de residuos sólidos generados en pequeñas y medianas instalaciones turísticas hoteleras cubanas. Aplicación instalaciones turísticas hoteleras de Villa Clara”. Tesis para optar al grado de Master Universidad Central "Marta Abreu" de Las Villas. 2013. URL: http://catedragc.mes.edu.cu/repositorios/

[26] M. Cazull Imbert. "Gestión de la transferencia de tecnología en la industria cubana del reciclaje: método y procedimientos". Tesis para optar al grado de Doctor. Universidad Central "Marta Abreu” de Las Villas, 2008. http://catedragc.mes.edu.cu/repositorios/

[27] F. Evia Lanier. "Metodología de diseño de la cadena de suministro inversa. Una contribución a la logística reversa". Tesis para optar al grado de Doctor. Instituto Superior Politécnico "José Antonio Echeverría", 2008. URl: http://catedragc.mes.edu.cu/repositorios/
[28] C. Toledo Hernández. "Modelo de gerenciamiento da logística reversa integrado às questões estratégicas das organizações". Universidad de Estadual Paulista: Faculdad de Engenharia do Campus, pp. 17-142. 2010. URL: http://catedragc.mes.edu.cu/ repositorios/

[29] M.V. de la Fuente y L. Ros. "Aplicación de Redes de Petri para la modelización de procesos en Logística Inversa". 3rd International Conference on Industrial Engineering and Industrial Management XIII Congreso de Ingeniería de Organización. September 2nd-4th, Barcelona-Terrassa, 2009. URL: www.adingor.es/congresos/ web/uploads/cio/cio2009/466-475.pdf

[30] L.D. Murillo Soto. "Redes de Petri: Modelado e implementación de algoritmos para autómatas programables". Tecnología en Marcha. Vol. $21 \mathrm{~N}^{\circ}$ 4, pp. 102. 2008. URL: revistas.tec.ac.cr/index.php/tec_marcha/ article/view/231 